\title{
Du diagnostic à la réhabilitation des barrages anciens
}

\author{
G. Degoutte, P. Royet \\ CEMAGREF, groupement d'Aix-en-Provence, B.P. 31, 13612 Aix-en-Provence Cedex 1
}

\section{Introduction}

Depuis le début du XIX ${ }^{\mathrm{e}}$ siècle, de nombreux barrages destinés à l'alimentation en eau potable ont été construits en France.

Contrairement aux ouvrages de production hydroélectrique ou aux barrages destinés à la navigation qui sont gérés par des maîtres d'ouvrage spécialisés possédant de nombreux ouvrages et disposant de larges compétences techniques en la matière, les barrages destinés à l'alimentation en eau potable sont la propriété de maîtres d'ouvrages très dispersés et non spécialisés : communes, syndicats intercommunaux, départements.

Cette particularité est évidemment d'une grande importance pour le contrôle et l'entretien de ces ouvrages, d'autant que leur dimension souvent moyenne fait qu'ils retiennent moins l'attention que les très grands barrages.

\section{Pourquoi un diagnostic?}

Un barrage ancien, par exemple centenaire, présente l'avantage d'avoir été testé suffisamment longtemps pour être considéré comme ayant fait ses preuves. $\mathrm{Si}$ de plus l'aspect général est bon, on est naturellement enclin à ne pas s'inquiéter : le barrage fait partie du paysage. Mais, ce sentiment de sécurité n'est pas toujours fondé:

1.1. La crue maximale connue par l'ouvrage peut être très inférieure à la crue de projet : le barrage n'a pas alors été vraiment testé dans les conditions les plus défavorables.

Ces risques sont assez évidents si l'on parle d'un barrage en remblai dont l'évacuateur de crue s'avère insuffisant. Mais c'est aussi très grave dans le cas d'un barrage poids déversant: les moments des forces tendant au renversement de l'ouvrage croissent comme le cube de la hauteur. Pour les petits et moyens barrages, ce critère est prépondérant.
Ainsi un barrage en maçonnerie construit en 1877 a dû être conforté en 1984. Un calcul moderne avait montré qu'il était stable selon les critères actuels tant que le débit n'excédait pas $450 \mathrm{~m}^{3} / \mathrm{s}$. Or la plus grosse crue connue en un peu plus de 100 ans était du même ordre $\left(420 \mathrm{~m}^{3} / \mathrm{s}\right)$ et la crue millénale avait été estimée à $850 \mathrm{~m}^{3} / \mathrm{s}$ [DEGOUTTE, MONCHALIN, 1986].

1.2. Un barrage peut avoir résisté à un certain événement, par exemple une crue donnée, ou une vitesse de vidange donnée..., et ne pas résister au même événement une seconde fois: c'est par exemple le cas d'un barrage en maçonnerie qui aura résisté à une forte crue qui tendait à provoquer son renversement, en créant en son sein des voûtes de décharges.

1.3. Les maçonneries vieillissent sous l'effet des sollicitations prolongées tant hydrauliques que mécaniques:

- lente dissolution des liants sous l'effet des percolations de l'eau; il en résulte à la fois une perte de poids de l'ouvrage, une augmentation des sous-pressions internes et une accélération des fuites;

- fatigue de la maçonnerie sous l'effet des déformations répétées dues aux variations thermiques et aux fluctuations du plan d'eau.

Ce vieillissement des maçonneries ne se détecte pas forcément du premier coup d'œil, car les vieux ouvrages d'art en maçonnerie présentaient des parements d'excellente qualité mais un remplissage plus médiocre.

Ainsi un barrage en maçonnerie construit en 1923 avait un excellent aspect. Des sondages réalisés en 1986 ont montré que la maçonnerie avait perdu une grande partie de son liant portant la densité de 2,4 à 2,25. Les sous-pressions dans le corps de l'ouvrage étaient élevées car le parement aval était très bien jointoyé.

1.4. Les remblais vieillissent aussi. Des phénomènes d'érosion interne peuvent lentement colmater des drains à granulométrie incorrecte et provoquer la saturation du parement aval. Une rupture par glissement de ce talus ou par renard peut alors survenir. 
Ainsi un petit barrage du Sud-Ouest a été détruit par renard en 1976. Ce barrage avait été construit 25 ans auparavant, vraisemblablement sans étude ni contrôle de mise en auvre.

Autre exemple, un barrage en arènes granitiques a vu son drain vertical se colmater 6 ans après sa mise en eau. Puis, 4 plus tard, son talus aval s'est saturé et a commencé à glisser [MONFORT et al., 1991].

Plusieurs dizaines d'années de comportement apparemment normal ne mettent donc pas à l'abri d'un risque de rupture d'un barrage en terre.

1.5. Enfin, les fondations vieillissent. En particulier les drains forés dans la roche de fondation peuvent se colmater peu à peu, par des apports de minéraux ou des dépôts organiques (bactéries, algues).

Ce cas a été rencontré sous l'appui central d'un barrage double voûte construit en 1972 en site granitique. Une reconnaissance des drains par caméra vidéo puis par diagraphie aux rayons gamma a permis de déceler un colmatage important des fissures, limité essentiellement au voisinage immédiat du forage [ROYET et al., 1990].

\section{Techniques de reconnaissance d'un barrage ancien}

\subsection{Les barrages en terre}

Les techniques de reconnaissance classiques sont les suivantes :

- tranchées de reconnaissance à la pelle mécanique en crête ou en pied de parement, à réaliser barrage vide ;

- sondages carottés réalisés depuis la crête et descendus dans la fondation;

- essais in situ permettant de mesurer des paramètres mécaniques interprétables : pénétromètre statique, pressiomètre, phicomètre ;

- essais de mécanique des sols sur échantillons intacts ou remaniés.

Il est important de valoriser au mieux les essais coûteux, c'est-à-dire les sondages carottés. La mise en place de piézomètres ou de capsules de pression interstitielle doit donc être envisagée pour apporter des renseignements utiles tant pour la reconnaissance que pour l'auscultation ultérieure.

Une nouvelle technique de reconnaissance est celle de la thermographie infrarouge pour détecter les zones les plus froides créées par l'eau à l'arrivée sur le parement aval des barrages.

\subsection{Les barrages en maçonnerie}

La reconnaissance est basée essentiellement sur des sondages carottés.
Outre l'observation des carottes, des prélèvements d'échantillons intacts permettent de faire des mesures de densité et de résistance mécanique. La mesure de la densité est particulièrement importante, car ce paramètre joue un rôle primordial dans la stabilité. Malheureusement, lorsque le corps d'un barrage en maçonnerie est poreux, le carottage désorganise le matériau prélevé et la mesure en laboratoire de la densité devient délicate.

Il est classique de profiter des sondages pour réaliser des essais d'eau de type Lugeon qui permettent d'apprécier la perméabilité du corps de l'ouvrage, de sa fondation ou de leur contact. Ces forages peuvent être ensuite utilisés comme piézomètres. D'autres techniques moins classiques permettent de profiter de la réalisation des sondages. Ainsi, la microsismique consiste à placer des capteurs dans un sondage et à émettre des vibrations soit en tête de sondage, soit sur un parement. Le profil de vitesses sismiques qui en résulte donne une indication précieuse sur la qualité des matériaux et sur la présence d'éventuelles zones plus faibles. Il permet en outre d'extrapoler les résultats ponctuels des sondages et d'indiquer s'il est nécessaire de pratiquer d'autres sondages.

Les techniques de diagraphies, issues de l'expérience pétrolière, enregistrent divers paramètres physiques des terrains traversés par un forage en fonction de la profondeur. L'interprétation de ces paramètres permet de renseigner sur les perméabilités, la densité...

La tomographie consiste à cartographier un paramètre, vitesse sismique par exemple, à partir de mesures réalisées entre deux sondages ou entre un sondage et un parement.

En résumé, au-delà de la technique lourde du sondage carotté, il existe une grande variété de techniques légères et performantes, faisant appel à des équipes très spécialisées. Le CEMAGREF a déjà expérimenté avec succès ces techniques originales: micro-sismique sur trois barrages, diagraphie par rayonnement gamma sur deux autres barrages.

\section{Le diagnostic}

Le diagnostic consiste à porter un jugement sur la sécurité de l'ouvrage. Il comporte toujours une étude hydrologique et un calcul de stabilité. Si des données d'auscultation sont disponibles, le diagnostic comprend également une analyse du comportement de l'ouvrage.

\subsection{Les barrages en terre}

Les calculs de stabilité nécessitent la connaissance :

- des densités en place des matériaux du remblai et de la fondation tant pour les zones saturées que pour les zones non saturées;

- des caractéristiques mécaniques à long terme obtenues par des essais triaxiaux sur échantillons intacts après consolidation ; 
- de la position de la ligne de saturation et des affleurements éventuels sur le parement aval;

- si possible, des pressions interstitielles.

Les calculs classiques de rupture circulaire, selon la méthode Bishop par exemple, sont très généralement suffisants. La difficulté n'est pas de réaliser ce calcul, mais bien de déterminer les paramètres nécessaires.

Des méthodes plus lourdes peuvent s'avérer utiles dans des cas difficiles : ce sont des calculs par éléments finis. Les codes de calcul existent, mais en petit nombre si l'on cherche à simuler de manière satisfaisante le comportement élastoplastique d'un sol. Ces méthodes peuvent être cependant intéressantes si l'on dispose de nombreuses données d'auscultation (piézométrie, pressions interstitielles, déformations).

\subsection{Les barrages en maçonnerie}

Le calcul de stabilité simple consiste à considérer une tranche verticale du barrage et à effectuer un calcul de résistance des matériaux, selon les hypothèses classiques de Navier. On vérifie la stabilité au glissement ainsi que l'absence de tractions.

Lorsque ce type de calcul montre que la stabilité est critique, on réalise généralement un calcul aux éléments finis. Ce calcul nécessitant la connaissance des modules d'élasticité de la maçonnerie et de la fondation, les reconnaissances préalables doivent avoir été orientées en ce sens.

\subsection{Conclusion du diagnostic}

Les résultats de la reconnaissance et des calculs peuvent déboucher sur trois types de recommandations :

- confortement du barrage ;

- baisse du niveau d'exploitation ;

- renforcement de la surveillance de l'ouvrage.

La troisième recommandation correspond au cas où le diagnostic permet d'écarter tout risque grave à court terme, mais laisse subsister des inconnues significatives. L'auscultation renforcée du barrage pendant quelques mois ou quelques années sera orientée dans le but de lever si possible ces inconnues et de déceler toute évolution inquiétante dans le comportement de l'ouvrage.

\section{Confortement des barrages}

Les techniques de confortement des barrages existants sont nombreuses. Nous nous limiterons, au moyen de deux exemples, au traitement des défauts les plus courants, c'est-à-dire :

- le manque d'étanchéité d'un barrage en terre;

- le manque de poids d'un barrage en maçonnerie.
Bien entendu il peut aussi être nécessaire de traiter l'étanchéité d'un barrage en maçonnerie, d'améliorer la stabilité d'un parement de barrage en terre, de renforcer l'étanchéité de la fondation ou d'agrandir l'évacuateur de crue...

\subsection{Renforcement de l'étanchéité d'un barrage en remblai : exemple du barrage des Sapins}

Le barrage des Sapins situé dans le département du Rhône est un remblai pseudohomogène en arènes granitiques de $16 \mathrm{~m}$ de hauteur au-dessus du terrain naturel. Le dispositif de drainage est constitué d'un drain cheminée de $10 \mathrm{~m}$ de hauteur et d'un drain-tapis au contact fondation-remblai sous la partie aval du barrage.

Construit en 1977-78, l'ouvrage était équipé dès l'origine d'un dispositif d'auscultation (mesure de débits, cellules piézométriques, topographie) dont les mesures étaient régulièrement faites mais jamais analysées.

Dix ans après la première mise en eau, lors d'une visite de routine, des désordres ont été constatés sur la partie inférieure du talus aval (glissements superficiels, venues d'eau). Leur aggravation rapide a amené à décider une vidange de la retenue.

L'analyse des mesures du dispositif d'auscultation, complétée par des reconnaissances légères par tranchées, a conclu à un colmatage du dispositif interne du remblai. Ces phénomènes auraient pu être mis en évidence plusieurs années auparavant par l'analyse des mesures d'auscultation. Avec le temps, la piézométrie est venue affleurer le talus aval provoquant alors des glissements superficiels et l'amorce de renards. La ruine de l'ouvrage était probable à court terme, si la vidange n'avait été rapidement entreprise.

La réparation a consisté à restaurer l'étanchéité du remblai et de la fondation. Le remblai a été traité par une paroi moulée souple au coulis autodurcissant et la fondation a fait l'objet de traitements ponctuels par injections. Par ailleurs la stabilité du talus aval a été légèrement améliorée par une recharge d'enrochements en pied et le dispositif d'auscultation a été complété par des piézomètres supplémentaires.

Le barrage est resté vide pendant seulement 4 mois d'hiver, à une période de l'année non primordiale compte tenu de sa vocation touristique.

\subsection{Confortement d'un barrage en maçonnerie à profil insuffisant : exemple du barrage de la Bourne}

Lorsque la stabilité d'un barrage-poids s'avère insuffisante, on peut envisager :

- d'augmenter le poids directement par ajout d'un massif de béton à l'amont ou à l'aval ;

- d'augmenter le poids indirectement par la réalisation d'ancrages actifs (tirants précontraints);

- d'améliorer la tenue au glissement par des ancrages passifs ;

- de diminuer les sous-pressions par amélioration de l'étanchéité et/ou par drainage. 
Le barrage de la Bourne, situé dans le département de la Drôme, est un ouvrage poids en maçonnerie de $18 \mathrm{~m}$ de hauteur sur fondation construit en 1877. Entièrement déversant, il présente un fruit aval de 0,57 , ce qui est très faible pour un tel type d'ouvrage.

Un diagnostic approfondi de ce barrage a été entrepris il y a environ 10 ans. L'étude approfondie des archives et des reconnaissances pour sondages carottés et géophysiques ont permis de conclure que :

- le corps de l'ouvrage était globalement en bon état ;

— la stabilité d'ensemble était assurée jusqu'à un débit sur le déversoir de $450 \mathrm{~m}^{3} / \mathrm{s}$.

Or la crue millénaire est estimée à $850 \mathrm{~m}^{3} / \mathrm{s}$ et la plus grosse crue connue à $420 \mathrm{~m}^{3} / \mathrm{s}$ (1986). Il a donc été jugé indispensable de conforter l'ouvrage.

La limitation de la charge hydraulique par agrandissement du déversoir était quasi impossible vu la configuration des lieux. Il ne restait dans ce cas que la solution de l'augmentation directe ou indirecte du poids. Les deux options ont été étudiées pour aboutir au choix des tirants précontraints, car cette solution présentait l'avantage de pouvoir être exécutée sans vidange du barrage.

Vingt-neuf tirants ont été réalisés, espacés en partie centrale de $2 \mathrm{~m}$ et précontraints à 50 tonnes. Il s'agit de tirants à double gaine scellés en profondeur et libres en partie supérieure, que l'on peut au besoin remettre en tension. La crête déversante, à l'origine en maçonnerie, a été remplacée par une poutre de répartition en béton armée en forme de seuil Creager.

Par ailleurs, des essais d'eau réalisés lors des forages d'installation des tirants ont permis d'identifier des zones de maçonnerie plus poreuse qui ont été traitées par injection avant pose des tirants.

La pose d'une cale dynamométrique sur l'un des tirants permet une auscultation à long terme du confortement de l'ouvrage.

\section{L'auscultation des barrages existants}

Il est maintenant tout à fait admis que les barrages neufs doivent être équipés de dispositifs d'auscultation. Par contre, il serait très dangereux de penser que l'on peut se dispenser d'ausculter un barrage ancien au seul bénéfice de l'âge.

On a vu sur les exemples précédents que les barrages subissent des processus lents de vieillissement qui peuvent à terme conduire à leur ruine. L'installation ou la remise à niveau d'un dispositif d'auscultation doit être conçue, pour un barrage ancien, dans le double but:

- de fournir les bases d'un diagnostic de l'ouvrage selon les méthodes de calcul modernes;

- de renseigner sur les évolutions futures de l'ouvrage.

Bien entendu, ces deux objectifs relèvent des mêmes types d'instrumentation, même si dans le premier cas on s'intéresse plus aux valeurs absolues des mesures, et dans le second cas à leurs variations.

\subsection{Les barrages anciens en terre}

Sauf mouvements visibles, on ne s'intéressera pas aux mesures de déformation sur un barrage ancien en remblai, pour lequel les phénomènes liés au tassement sont a priori terminés. Par contre les mesures hydrauliques sont primordiales.

La mesure du débit de fuite est indispensable car elle renseigne sur l'efficacité du barrage. Les écoulements sont collectés par un caniveau équipé à son débouché d'un seuil de mesure. Chaque fois que cela est possible, on s'efforce de mesurer séparément les écoulements en provenance du remblai, de la fondation et des appuis.

Les mesures de pression sont plus ponctuelles mais moins influencées par des phénomènes parasites. Deux types d'appareils sont utilisables:

- les piézomètres à tube ouvert mesurent la pression dans une certaine tranche verticale du remblai (la partie crépinée du forage); leur temps de réponse est variable selon la nature du matériau de remblai ;

- les cellules de pression fournissent une mesure ponctuelle, leur temps de réponse est très court ; elles peuvent être mises en place à différentes cotes dans le remblai ou sa fondation, au moyen de forages.

On favorisera le contrôle de l'ouvrage en réalisant un profil d'auscultation rive à rive et non pas quelques profils amont-aval comme cela est fait plus classiquement sur les barrages neufs.

\subsection{Les barrages poids en maçonnerie}

Du fait de la rigidité globale de l'ouvrage, des mesures de déformations permettent de déceler un début de rupture par glissement ou renversement. On peut citer quatre types de dispositifs pour mesurer les déformations :

- les visées topographiques de cocardes scellées sur le barrage; il s'agit d'un dispositif peu coûteux à l'installation, permettant une bonne densité des points de mesures; par contre les campagnes de mesures, vu leur coût, ne peuvent être très fréquentes ; une précision de $0,5 \mathrm{~mm}$ peut être considérée comme bonne ;

- les pendules inversés placés dans un forage réalisé en crête du barrage ; ce dispositif plus coûteux que le précédent à l'installation s'avère plus économique en exploitation, mais surtout il permet des mesures fréquentes et très précises (de l'ordre de $0,1 \mathrm{~mm}$ );

- les clinomètres à silice scellés sur le parement aval ; très précis et peu encombrants, ils fournissent des valeurs ponctuelles de rotation;

- les vinchons et les fissuromètres scellés au droit d'une fissure ou d'un joint actifs.

Les mesures hydrauliques sont également précieuses. Comme pour les barrages en terre, il s'agit de piézomètres et de dispositifs de mesure des débits de fuite (globalement ou drain par drain). 


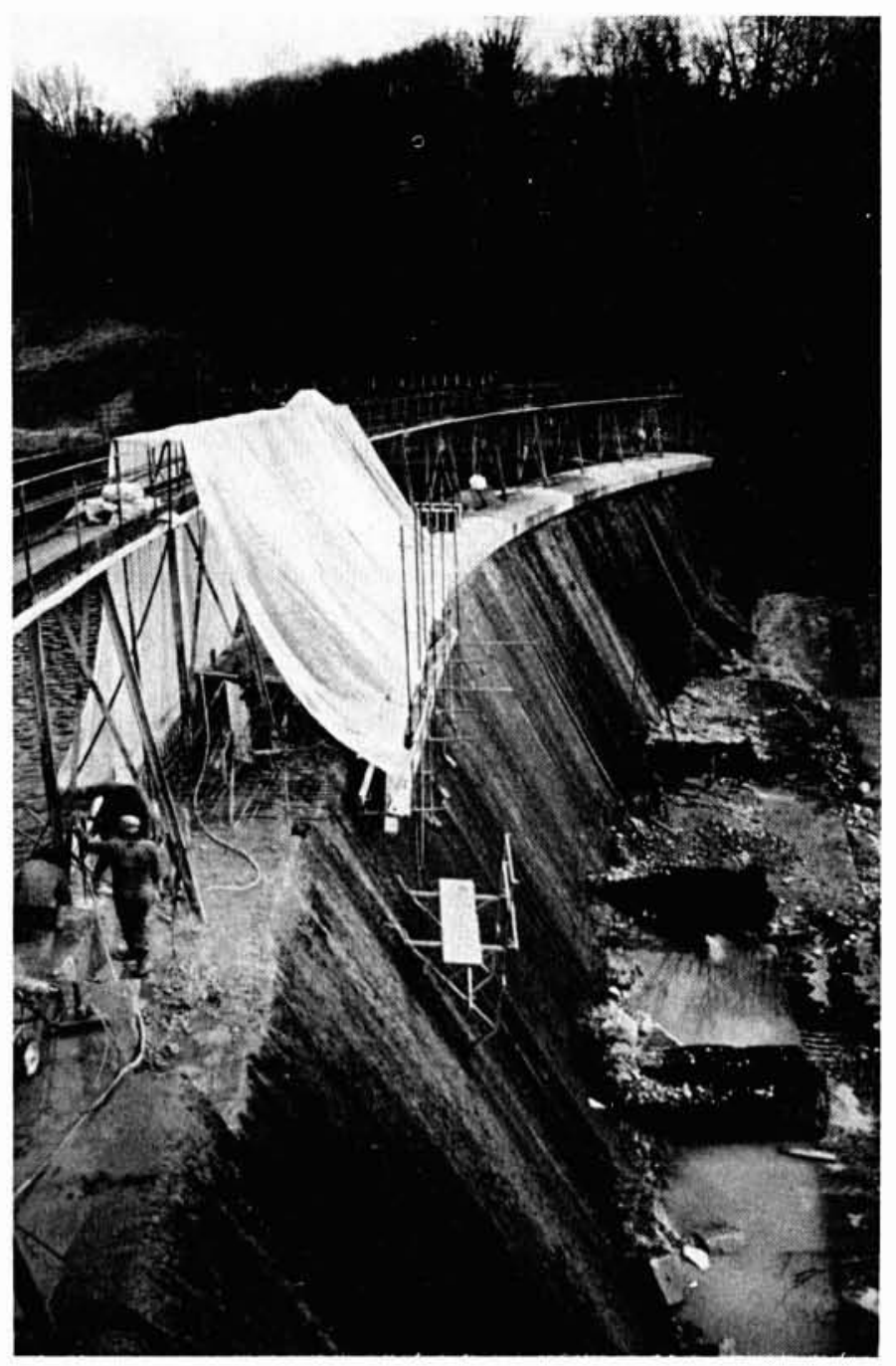

Barrage de la Bourne (26).

Vue générale des travaux de confortement.

De gauche à droite :

- tirant en attente.

- dérasement du couronnement,

- ferraillage et bétonnage de la poutre (sous la bâche),

- forage des drains (en pied aval).

Barrage de Saint Martin de Londres (34). Sondages de reconnaissance réalisés à partir d'une plate-forme posée sur la crête arrondie du barrage.

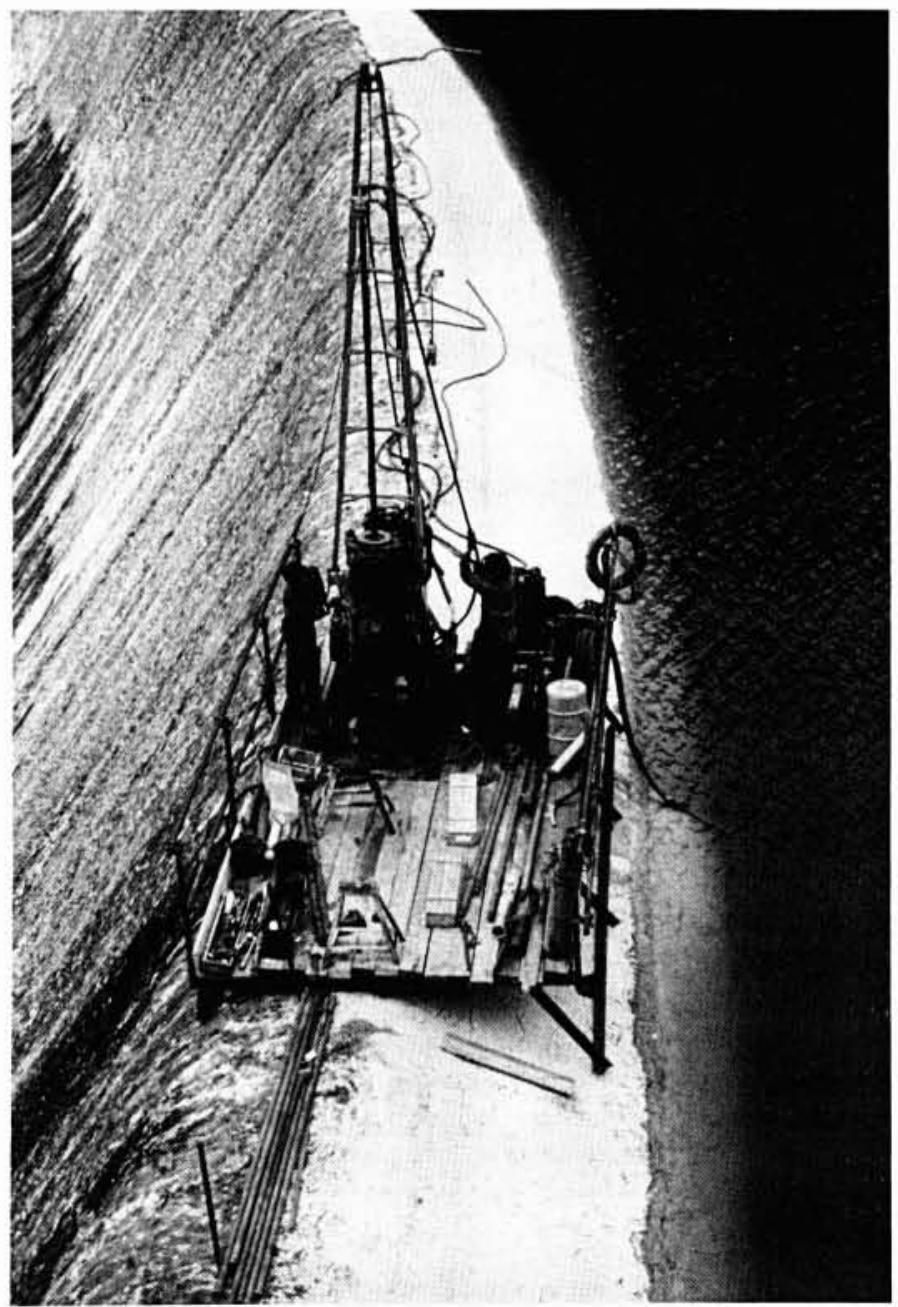




\subsection{Suivi des mesures d'auscultation}

Bien entendu, la pose d'appareils de mesure n'est pas une fin en soi : les mesures doivent être effectuées régulièrement et exploitées rapidement. La plupart des mesures peuvent être automatisées et on peut mettre des dispositifs automatiques d'alerte sur certains appareils, judicieusement choisis.

Les résultats d'auscultation font l'objet de deux traitements. L'un est de type statistique : au moyen de logiciels adaptés, il permet de mettre en évidence l'effet irréversible d'une série de mesures après avoir retiré les influences saisonnière et hydrostatique. Généralement on analyse l'ensemble des mesures d'auscultation tous les deux ans, et l'on vérifie régulièrement que chaque lecture, débarrassée de ses effets réversibles, ne présente pas une évolution nouvelle.

L'autre traitement est de type déterministe : il compare les déplacements, débits, pressions calculés à ceux qui sont observés pour les mêmes sollicitations extérieures.

Bien entendu, le jugement de sécurité de l'ouvrage ausculté est basé sur ces calculs, mais l'ingénieur s'appuiera aussi sur sa connaissance des cas semblables, c'est-à-dire sur son expérience.

\section{Diagnostic et auscultation: bases d'une bonne gestion}

Comme tout ouvrage, les barrages ont une espérance de vie, bien sûr difficilement chiffrable dans l'absolu, mais que l'on peut largement repousser par divers moyens.

Tout d'abord par une bonne connaissance de l'ouvrage, ce qui nécessite une mémoire vivante, qu'il y a lieu de stimuler de temps à autre. C'est l'objet du diagnostic qui sera d'autant plus lourd que la mémoire sera affaiblie.

Ensuite par un suivi régulier de l'état de santé, basé sur des visites régulières et une auscultation adaptée à l'ouvrage et à ses points sensibles. Les mesures, désormais largement automatisables, doivent être interprétées par des spécialistes.

Enfin par l'administration de remèdes appropriés, dès qu'apparaissent les premiers symptômes de désordres.

Le respect de ces principes de base permettra de disposer d'un ouvrage "bon pour le service ", c'est-à-dire capable en toute sécurité de contenir de l'eau pendant une longue durée de vie.

\section{Remerciements}

Les travaux du CEMAGREF sont encouragés par le Ministère de l'Agriculture - Direction de l'Espace Rural et de la Forêt et celui de l'Environnement - Service de la Recherche des Etudes et du Traitement de l'Information sur l'Environnement et Direction de l'Eau.

\section{Bibliographie}

Degoutte G., Monchalin G. (1986). - Renforcement d'un barrage par des tirants actifs. Informations Techniques du CEMAGREF, cahier $62 N^{\circ} 3,6$ pages.

Degoutte G. (1988). - Auscultation des barrages - Cours ENGREF et ENITRTS, CEMAGREF, Aix-en-Provence, 41 pages.

Royet P., Degoutte G., Becquart D. (1990). - Diagraphies et diagnostic de l'évolution des débits drainés sous un barrage voûte. Journées d'études diagraphies et mécanique des terrains, Bordeaux, pages 273-287.

MONFORT L. et al. (1991). - Eléments de méthodologie pour la détection et l'analyse du vieillissement illustrés par des exemples. XVIF Congrès CIGB, Q.65, R.23, pages 405 à 437.

Degoutte G. (1992). - Guide pour le diagnostic rapide des barrages anciens. CEMAGREF, série Hydraulique Agricole, $N^{\circ} 13,100$ pages.

Barrage des Sapins (69) - Réalisation d'une paroi moulée sous coulis à la benne à câble (Photo CEMAGREF).

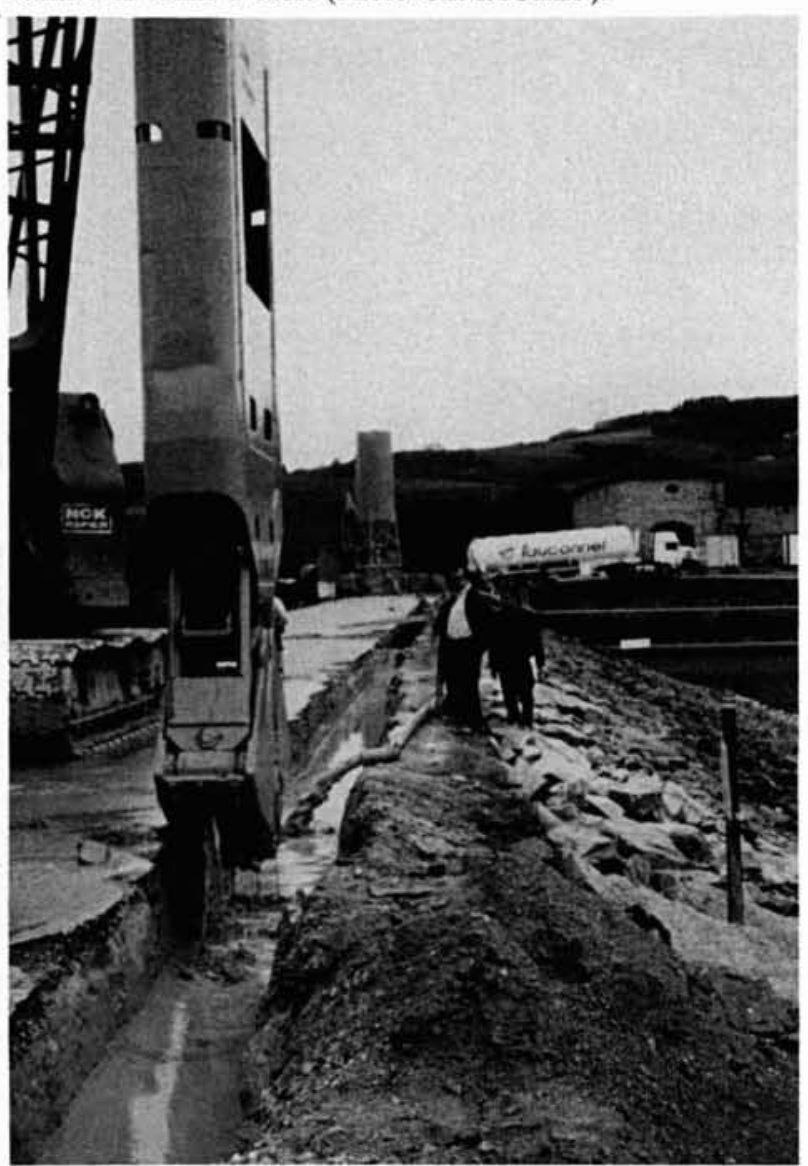

